\title{
Diagnóstico del estado de la alfabetización en información (ALFIN) en las universidades chilenas
}

Miguel Ángel Marzal

Doctor y Profesor Titular del Departamento de Biblioteconomía y Documentación de la universidad Carlos III de Madrid - España

Elvira Saurina

Máster y Subdirectora Área Ciencias y Tecnología,
Sistema de Bibliotecas de la Pontificia
Universidad Católica de Chile

http://dx.doi.org/10.1590/1981-5344/2070

Se presenta la fundamentación teórica, metodología, aplicación de encuestas, análisis y discusión de resultados de un proyecto de trabajo de campo para hacer un diagnóstico sobre la implantación o posibilidades de desarrollar programas de alfabetización en información en universidades chilenas. Se analiza el planteamiento del proyecto: la fundamentación epistemológica, estableciendo las convenciones de lo que se va a entender por alfabetización en información, las competencias y su proyección en programas educativos mediante un modelo (Tuning), como también en el currículo, y por último las competencias en información para definir el diseño instruccional y el diseño curricular de los programas de alfabetización en información. Se presenta la metodología del proyecto para conocer el estado del arte de estos programas en las universidades chilenas, formulando una encuesta que fue aplicada, de modo que se presentan y analizan los resultados, a partir de los cuales se enuncian unas recomendaciones, que sirven para apoyar una vía que establezca un modelo de integración de los programas de alfabetización en información para la educación en universidades.

Palabras clave: Alfabetización en información; Competencias informacionales; Encuestas: Modelo educativo competencial; Tipos de competencias; Programa educativo de alfabetización en información. 


\section{Diagnosis of the state of information literacy (IL) in Chilean universities}

This article outlines the theoretical foundations, methodology, survey findings, analysis and discussion of results of a fieldwork project to assess the implementation of information literacy programs at Chilean universities. The project approach is analyzed: the epistemological foundations, the conventions of what is understood for information literacy and competencies, and their projection in educational programs using a model (Tuning), as well as in the curriculum; and finally the analysis of information competencies to define instructional and curriculum design of information literacy programs. The methodology used to know the state of the art of these programs at Chilean universities includes the survey that was applied and the analysis of its results, from which recommendations were drawn to support a way to establish a model for the integration of information literacy programs within university curricula.

Keywords: Information Literacy; Informational Competencies; Questionnaire; Educational Model of competencies; Type of competencies; Educational Program of information literacy.

Recebido em 08.04.2014 Aceito em 06.05.2015

\section{Introducción}

El desarrollo de un trabajo de investigación de campo (llamémoslo investigación aplicada) requiere, inicialmente, del diseño de un plan metódico a partir de la formulación de una hipótesis y considerando siempre unas "convenciones epistemológicas", para evitar incurrir en un debate académico científico, conceptual y terminológico, sumamente interesante pero que puede entorpecer la consecución de unos resultados de investigación (Ilamémosles deliveries), mediante el cumplimiento de unos "hitos" en el proceso de investigación (Ilamémosles milestones), esto es, definir los fundamentos de la investigación de base, los principios que regirán el desarrollo metodológico de la investigación.

De este modo, se planteó un proyecto de investigación en el área de las competencias informacionales, en el mes de octubre del año 2012, por parte del Sistema de Bibliotecas de la Pontificia Universidad Católica de Chile, con el fin de conocer el estado del desarrollo de las competencias informacionales o alfabetización en información (ALFIN) de las 
universidades públicas y privadas chilenas, con especial atención a terminología, definición, estándares y modelos, programa y actividades, niveles y modalidades de implementación, tipos y herramientas de evaluación para unas propuestas de mejora en unos programas de formación básicos en la Educación Superior del siglo XXI.

\section{Fundamentos epistemológicos de proyecto: convenciones en una investigación de base para una investigación aplicada}

Una vez que estaban definidos el campo, el objeto, objetivos e hipótesis del proyecto de investigación, se debía proceder a la fundamentación epistemológica del proyecto para su eficaz desarrollo:

\section{Convenciones para el campo y la hipótesis del proyecto}

El proyecto procedió a establecer unas convenciones para la correcta comprensión de sus objetivos, milestones y deliveries, sin el ánimo de un debate académico sino para un diseño de procedimiento metodológico:

a)La Formación de Usuarios será entendida como la acción formativa ejercida por bibliotecarios para que sus usuarios adquiriesen las destrezas y habilidades precisas, incluso idóneas, en un uso eficaz de los recursos, servicios de información, así como de la colección documental, en la biblioteca;

b)La diferencia notoria y notable entre alfabetización en información y competencias en información, a veces confundidas y en muchas ocasiones, especialmente en el ámbito de los profesionales en unidades de información, consideradas como potenciales sinónimos entre los que se debe elegir;

Situados en el ámbito de las competencias en la Educación para el siglo XXI no consideraremos, para este proyecto, la formación de usuarios. Necesitaremos, sin embargo, el establecimiento de unas convenciones para el desarrollo del proyecto: la diferenciación de alfabetización en información y competencias en información (WEBBER; JOHNSTON, 2003).

Conforme al desarrollo, formulación académica, proyectos e investigaciones respecto a la alfabetización en información, la consideraremos una especialidad académica dentro del área de conocimiento de Biblioteconomía y Ciencias de la Documentación. Basamos esta convención en los siguientes elementos:

I Su propio espacio científico, conocido desde las definiciones de ALA, CILIP, etc.: alfabetización en información es saber cuándo y por qué necesitas información, dónde encontrarla, y cómo evaluarla, utilizarla y comunicarla de manera ética, una 
definición que con ciertas variantes se repite en toda la comunidad científica.

II Su propia justificación e hipótesis: inclusión social y digital, desarrollo humano sostenible, comprensión por evaluación de contenidos mediante lectura y escritura digitales (por ello la denominación de "alfabetización").

III Su propio método, fundamentado en: el método científico (para el diseño de los programas educativos), las Normas (definen el qué del proyecto de alfabetización en información), los Modelos (definen el cómo de los proyectos).

IV Sus propios tópico, objeto de investigación y objetivos: competencias en información para conocer y saber en la Sociedad del conocimiento, mediante el aprendizaje permanente y colaborativo.

V Su propio campo de aplicación: bibliotecas digitales educativas y Web semántica

Desde la óptica del proyecto y sus convenciones la diferencia entre ambos conceptos, hace que el debate pueda ser inscrito en unos límites precisos: la alfabetización en información sería una especialidad cuyo tópico y objeto de investigación son las competencias en información.

\section{2 Convenciones para el objeto y objetivos del proyecto: el modelo educativo competencial}

El proyecto, contextualmente, se inscribe dentro de la tendencia al desarrollo e implantación, en Educación Superior, del Modelo Educativo Competencial, que en principio se orienta decididamente hacia el aprendizaje al estimular la capacidad de innovación y las potencialidades de los educandos. Esto no significa postergar la Educación, pues educar en competencias infiere una notoria innovación docente, si bien también es patente, consultando la literatura especializada, que este tipo de educación adolece de metodología apropiada, así como de fundamentos pedagógicos y didácticos.

Esta ausencia de fundamentos pedagógicos y didácticos ha determinado que, cuando se hizo preciso desarrollar un modelo formativo basado en competencias esencialmente por una exigencia económica, se propusiesen unos modelos más referidos al ámbito laboral que al educativo, como demuestran los tres modelos más relevantes, anotados en el Manual de Difusión del Modelo de Educación basada en competencias del subsistema de universidades tecnológicas (MEBCSUT) de la Coordinación General de Universidades Tecnológicas, en septiembre de 2008: 
a)Modelo alemán, basado en la idea de la cualificación laboral, por lo que se detenía en la regulación de las atribuciones de órganos de administración y empresas.

b)Modelo de Reino Unido y EEUU. En Reino Unido la competencia se definía respecto a la calidad en la función conforme a las normas necesarias para el trabajo. En EEUU la competencia no se analiza desde la perspectiva de las tareas en un oficio, sino desde las competencias que habilitan a una persona para ejercer ese oficio.

c)Modelo canadiense, consistente en capacitar a las personas para que asuman sus responsabilidades y desarrollar las competencias necesarias en función del mercado laboral presente y futuro, contribuyendo al desarrollo socio-económico del país.

d)Estos tres modelos habrían de tener una extensión, por comprensión de las competencias de forma más generalista y amplia (no sólo en el ámbito laboral) en el llamado Modelo Quebequés (al que pertenecen insignes investigadores del modelo competencial), por cuanto atiende a la necesidad de definir políticas en materia de educación competencial, que éstas se plasmen en programas de estudio, que deberán ser aplicados por docentes formados específicamente.

Uno de los hitos fundamentales en la conversión del modelo formativo competencial en modelo educativo competencial, fue la definición desde 1998 y su puesta en práctica (dependiendo de los países) entre 2005 y 2010 del Espacio Europeo de Educación Superior (EEES), cuyos objetivos declarados eran reestructurar las titulaciones (conforme a los nuevos desafíos del conocimiento), los niveles educativos (Grado, Postgrado, Doctorado), con el objetivo a una compatibilidad de grados académicos para lo que se impulsaba un ambicioso plan de movilidad de discentes y docentes, generar un sistema de evaluación de la calidad, asegurar el aprendizaje permanente entre los egresados $\mathrm{y}$, todo ello, lo que más importaba para nuestro proyecto, un sistema de créditos, los ECTS (European Credit Transfer System), destinado a medir la adquisición de conocimientos, capacidades y destrezas necesarias para el dominio competente de las funciones para las que ha sido preparado el estudiante en el Grado.

El modelo educativo competencial conlleva, en todo caso y ha de tenerse muy presente, un conjunto de transformaciones:

a)Respecto al educando, si bien la integración de las competencias implica la movilización de los conocimientos referidos a un contexto, el carácter individual de las competencias hace que en este modelo educativo sea imprescindible un análisis profundo de los estilos de aprendizaje de los educandos (ALONSO; GALLEGO, 2010).

b)Respecto al docente, según Perrenoud (PERRENOUD, 2004) la docencia debe dirigirse a organizar y estimular situaciones de aprendizaje, dirigir el progreso de aprendizaje e implicar a los alumnos en 
su proceso de aprendizaje, un uso eficaz de las TIC, junto con asumir con factor primordial la propia formación continua.

c)Respecto al sistema de evaluación del modelo, debe fundamentarse en la formación, promoción, certificación y mejora docente (RIAL, 2007).

d)Hasta aquí está expuesta la fundamentación teórica y formulación de principios del modelo educativo competencial, pero para su desarrollo en programas formativos y educativos, conforme a las necesidades de formulación de nuestro proyecto, se hace imprescindible la formulación de convenciones de trabajo:

\subsubsection{Las Competencias para programas educativos en competencias}

En muchas ocasiones, para la precisión de un concepto se hace necesaria una definición en negativo, es decir, lo que no es o no debe ser, de modo que se garantiza una mejor aplicación. En este sentido, es muy útil la exposición de $X$. Roegiers sobre los tres errores más comunes en torno al concepto de competencia (ROEGIERS, 2007):

a)La noción mecanicista de competencia como "conocer para actuar", una orientación hacia la producción de bienes y servicios;

b)La asociación de la competencia con un proceso de aprendizaje fragmentado, por identificar el aprendizaje por competencias con el aprendizaje por objetivos;

c)El descuido en evidenciar la estrecha relación entre competencia y evaluación

d)Son múltiples las definiciones de competencias, pero para su aplicación en programas educativos competenciales conviene considerar dos aspectos:

i La existencia de unos elementos de definición permanentes: se convierten en una característica permanente de la persona, se manifiestan al realizar con éxito una actividad, son causa de la eficacia y optimización laboral y son extrapolables a distintos entornos y contextos,

ii La definición de competencias parece responder a dos modelos (LUENGO; LUZÓN; TORRES, 2008): un primer modelo las define de forma individual y cognitiva (anglosajón), luego muy criticado por desatender la dimensión sociológica y colectiva; un segundo modelo social (francófono, M. Eraut) que distingue un concepto situado socialmente (capacidad para realizar tareas conforme a estándares) y situado individualmente (capacidades personales requeridas para una actividad),

iii Estos aspectos, convertidos en condicionantes y requisitos, han provocado la enunciación de unos enfoques para la aplicación de las competencias (MULDER; WEIGEL; COLLINS, 2007):

a)Enfoque conductista o behaviorista, defensor de unas competencias que se adquieren mediante la formación y se basan en la descripción de conductas observables, siendo sus caracteres la 
demostración, la observación y evaluación de comportamientos (McClelland),

b)Enfoque genérico, que identifica las habilidades estándar para luego, mediante análisis estadísticos de rendimiento, distinguir las personas más eficientes y sus caracteres más definitorios,

c)Enfoque cognitivo que prima la atención sobre los recursos mentales de los individuos al realizar tareas importantes, por lo que establece una íntima relación con las habilidades intelectuales y la inteligencia, de modo que utiliza modelos psicométricos sobre inteligencia y eficacia en el procesamiento de la información. Este enfoque se ha expandido hacia un modelo socio-constructivo, que entiende las competencias como factor necesario para una acción exitosa en sociedad, respecto al aprendizaje, solución de problemas, incertidumbre, procesamiento de la información, toma de decisiones, trabajo colaborativo.

El sesgo de estos enfoques ha hecho posible una tipificación de las competencias en: Competencia Laboral, relacionada con los procesos productivos por ser causa de una mayor eficiencia laboral; Competencia Profesional, relacionada con la consecución de grados académicos y orientada a la resolución de problemas; Competencia Educativa, sin duda la que debe atraer toda nuestra atención.

En Educación las competencias deben movilizar conocimientos, procedimientos y actitudes a partir de contenidos de aprendizaje para la toma de decisiones al enfrentar una hipótesis científica o un desafío profesional. De este modo, son sus propiedades primordiales (CARRERA; MARÍN, 2011): su movilización de "recursos", internos referidos a las potencialidades del educando (saber-hacer, saber-ser) y externos referidos a los materiales didácticos, recursos de infraestructura, la cualificación de los docentes; su carácter interrelacionado; su uso en la solución de problemas dentro de un contexto; su evaluación. La competencia educativa, pues, debe exigir al educando iniciativa, transferencia en el conocimiento e innovación.

\subsubsection{Definición de un marco programático para la aplicación de las competencias educativas}

Sin duda, este marco programático lo ha proporcionado el Proyecto Tuning, surgido en 2000 en conexión directa con el EEES, con el objetivo de proporcionar una base para la mejora de la calidad de la Educación Superior y unas herramientas destinadas a garantizar la cooperación y diálogo entre universidades, así como enunciar unas estrategias académicas para la consecución de unas competencias educativas mediante su aptitud y su adecuación a los fines planteados en los objetivos educativos del proyecto. 
Para el desarrollo del proyecto Tuning se planteó una metodología que, como demuestra la MEBCSUT, arriba citado, integraba dos fases:

1)Fase I, en la que su objetivo era la compatibilidad de los programas de estudio de los países miembros para mutuo reconocimiento académico, a partir de resultados de aprendizaje, expresados en competencias, genéricas y específicas, y representados en ECTS. En el proceso se consultó a empleadores, graduados, asociaciones profesionales, agentes sociales, docentes;

2)Fase II, cuyo objetivo era desarrollar medios para la mejora y calidad de Educación, mediante la identificación precisa de las competencias genéricas y luego las específicas, fortalecimiento de la función de los ECTS, desarrollo de enfoques educativos y de evaluación área la mejora de la calidad educativa. Llegados a este punto, Tuning desarrolló un modelo de diseño de planes de estudio, con sus etapas: cumplimiento de condiciones básicas; definición de perfil conducente al título; descripción de objetivos; identificación de competencias genéricas (instrumentales, interpersonales, sistémicas) y específicas; traducción de contenidos al plan de estudios; traducción a unidades y actividades educativas; definición de enfoques educativos; desarrollo de sistemas de evaluación.

El proyecto Tuning es la historia de un éxito, en el sentido de que se ha convertido en un referente mundial y una plataforma de convergencia para la cooperación, interoperabilidad y calidad de Educación Superior a escala planetaria. El proyecto ha tenido su proyección en Rusia (http://www.tuningrussia.org/) ${ }^{1}$, dentro de Estados Unidos en los estados de Minnesota, Indiana y Utah (http://www.mhec.org/programs/tuning), las repúblicas centroasiáticas de la extinta URSS, la TuCAHEA (http://www.tucahea.org/), así como también una expansión en África (http://www.tuningafrica.org/). En Japón se ha publicado una nueva versión para dar difusión y conocimiento al Proyecto Tuning Europa. La vigencia y dinamicidad de Tuning queda patente, además, en que tiene como entidad gestora del proyecto una entidad, la Tuning Academy, que ha comenzado la publicación del Tuning Journal for Higher Education, desde noviembre de 2013 (http://tuningacademy.org/tuning-journal). De otro lado, se está desarrollando un proyecto, EU/US Research Project (http://unideusto.org/tuningeu/home/385-euus-research-project.html), financiado conjuntamente por la Comisión Europea y Lumina Foundation (EEUU), y cuyo marco de investigación es el modelo educativo basado en resultados del aprendizaje y competencias para el aprendizaje permanente. El objetivo es conocer cómo se está aplicando este modelo educativo en actitudes, procesos de trabajo, metodologías didácticas y evaluación en universidades, así como conocer la percepción de los agentes implicados

\footnotetext{
${ }^{1}$ Todos los documentos que se presentan con un enlace en este apartado 2.2.2., están disponibles en las direcciones electrónicas reseñadas. Todas ellas en Acceso 27 de mayo de 2015.
} 
en este modelo educativo, docentes, discentes, gestores. Para el análisis de estos fenómenos se ha elaborado una encuesta que se está aplicando en Europa y Estados Unidos.

Este mismo programa ha tenido un impacto innegable en América Latina como región o área geográfica conjunta, que planteaba iniciar el proyecto (http://tuning.unideusto.org/tuningal/) durante los años 2004-2007, y aún hoy muestra su vigencia al proyectarse como modo de innovación educativa y social durante los años 2011-2013 (http://www.tuningal.org/).

De acuerdo a la citada MEBCSUT, el Proyecto Tuning Latinoamérica parte de la constatación del avance de la globalización y la necesidad de compatibilizar programas de Educación Superior. La aspiración en América Latina surge en la IV Reunión de Seguimiento del Espacio Común de Enseñanza Superior de la UE, América Latina y Caribe (UEALC), en Córdoba (España), 2002. Se comenzó a preparar la propuesta y se presentó al Programa Alfa en 2003, por 8 universidades (entre ellas, la Universidad de Chile y Universidad Católica Andrés Bello). La idea era adaptar las Fases Iy II del Proyecto Tuning Europa, comenzando a trabajar 62 universidades latinoamericanas, 120 nuevas después.

El proyecto Tuning Latinoamérica tuvo cuatro fases: sensibilización y difusión; diálogo entre pares; implementación de las competencias (talleres, seminarios, etc.); internacionalización (calidad). Todo culminó con la presentación de referencias metodológicas para el desarrollo del modelo con la Reunión en México DF de "Tuning América Latina", en 2007, recomendando una imprescindible financiación para soportar estrategias metodológicas conjuntas con sus distintas modalidades, formación de profesores e interoperabilidad de titulaciones.

\subsubsection{Proyección del modelo en programas educativos y el currículo}

La aplicación concreta de un modelo educativo basado en competencias en programas educativos específicos precisó de un amparo pedagógico, que lo ha proporcionado el Constructivismo $y$, aún más, la Teoría de la elaboración sobre competencias, basada en el Constructivismo, que propone el diseño instruccional de secuencias didácticas a partir del análisis de contenido (organización de contenidos específicos de una materia) y análisis de tareas (jerarquía del aprendizaje, que debe ser ascendente desde las habilidades básicas a las más complejas).

La competencia, según se ha visto, moviliza un conjunto de conocimientos, actitudes y conductas, por esta razón un programa de formación debe contemplar un número reducido de competencias a lograr, cada una integrante de un elevado número de recursos. Por esta razón, en opinión experta de Tardif, los programas basados en competencias deben contemplar ocho etapas (TARDIF, 2003), planificando: las competencias; el grado de desarrollo esperado en cada competencia; recursos a movilizar; escalabilidad de las competencias; modalidades 
pedagógicas; modalidades de evaluación; organización del trabajo de docentes y discentes; modalidades de apoyo en los aprendizajes.

Los programas buscan su plasmación en un currículo. Un currículo define las grandes orientaciones de un sistema educativo (JONNAERT et al., 2006), por lo que debe contemplar los parámetros sociales que condicionan el sistema educativo, siendo los principales parámetros: el nuevo concepto de conocimientos e inteligencia, determinado por el modo de acceder a la información; la demanda social, pues los empleadores buscan que los profesionales conciban global y totalmente las situaciones a las que enfrentarse; la revolución digital, por el volumen inconmensurable de datos e información.

Se impone, pues, un enfoque del currículo cada vez más diferenciado de planes y programas de estudio, por cuanto están cambiando los contenidos y métodos didácticos. A este respecto, es muy atractiva la recomendación de la Secretaría de Educación de México (SEP, 2009), citada por García Retana (GARCÍA RETANA, 2011), según la cual el currículo debe integrar la consecución de unas categorías de competencias básicas: competencias para el aprendizaje permanente; competencias para el manejo de información; competencias para el manejo de situaciones; competencias para la convivencia; competencias para la vida en sociedad.

\section{Delimitación del objeto de investigación: las competencias en información para programas de alfabetización en información}

Si las competencias se han proyectado con extraordinaria eficacia en el currículo y en Educación, la aparición de Internet $y$, muy pronto, el decidido avance hacia la Web del Conocimiento y Semántica, extendían la imperiosa necesidad de un nuevo modo de aprender en Web (del elearning al m-learning), unas nuevas estrategias didácticas que desembocaban en la especialidad de Tecnologías Educativas, un nuevo modo de leer y escribir en digital (una alfabetización digital y múltiple) y un nuevo método de convertir la información digital ingente en conocimiento mediante una categoría específica de competencias, arriba indicada, las competencias en el manejo de información, base de un nuevo modo de apoyar el método científico en los nuevos entornos, como acredita el excelente Modelo Gavilán, en su Competencia para Manejar Información (Disponible en: <http://www.eduteka.org/modulos/1>. Acceso: 27 mayo 2015).

La proyección de las TIC en Educación supuso el origen de impulsar las aptitudes y capacidades tecnológicas, encargadas a la alfabetización digital, pero planteando la necesidad inexcusable de comprender, conocer y saber en Web, una lecto-escritura digital que reclamaban competencias en el discurso electrónico. La combinación progresiva de informaciónconocimiento-saber consolidaría el binomio "competencias" e 
"información". Para su correcto desarrollo surgiría la educación en competencias, que proporciona al educando "competencias interpretativas" (destreza en hallar significado a la información), "competencias argumentativas" (capacidad de razonamiento expositivo al negociar la construcción de conocimiento y durante su comunicación), "competencias propositivas" (capacidad de formular propuestas para generar un nuevo conocimiento). Así se irían definiendo las competencias informativas, cuyos objetivos eran facultar conocimientos y habilidades en la gestión de la información, en el uso y aplicación de los contenidos, en la edición y comprensión del conocimiento obtenido.

C.S. Bruce (BRUCE, 1997) plantearía, más tarde, la noción de las competencias informacionales como un proceso compuesto de siete fases (caras), a modo de peldaños, que van ejercitando a las personas desde la captación de información a la obtención de conocimiento y saber. Las competencias en información situaban a los educandos en un proceso piramidal y ascensional de aprendizaje, que desde la base a la cúspide va desarrollando destrezas (aptitud manipulativa), luego habilidades (capacidad de automatizar y optimizar procesos dominados por las destrezas), para llegar a las competencias (actitud para aplicar el "conocimiento" en la resolución de problemas concretos mediante el "saber").

Las competencias en información, según hemos ya apuntado, por su indudable relevancia (de un lado como pilar de la innovación, de otro como instrumento contra las brechas digitales) se convertían en objeto de investigación de la alfabetización en información, como especialidad académica. Por su propia dinámica, pues, la alfabetización en información se ha proyectado en sus propios programas competenciales, con la finalidad de obtener una "materia didáctica". Los programas de alfabetización en información deben, en nuestra opinión, presentar una estrategia didáctica apropiada, que, en nuestra opinión, presenta los siguientes caracteres (MARZAL; PARRA, 2010):

\section{a)Diseño instruccional}

Como conjunto de principios que fundamentan la "intención" y finalidad educativas. Parece muy adecuado que como contexto educativo, el diseño instruccional debe ser flexible, esto es, adaptado a las distintas circunstancias de intereses, ritmo y capacidad de aprendizaje de los educandos. El diseño instruccional, además, tiene que elegir unas modalidades instruccionales, que definen el programa:

Modalidad formativa, esto es, si el programa va a impartirse en Educación Informal (como un curso de iniciación o especialización), Educación No Formal (como cursos en línea, e-learning, m-learning, MOOC) o Educación Formal (como materia transversal, como materia de especialidad en un Grado o tópico de investigación en Postgrado o Doctorado). 
i Modalidad de difusión, presencial, semipresencial (blended learning) o en línea, para lo que es fundamental determinar la plataforma digital de aprendizaje.

ii Modalidad didáctica, referida al uso de materiales didácticos idóneos, que en el caso de estos programas, por sus propiedades, pueden ser tutoriales interactivos, materiales audiovisuales (uso didáctico de vídeos, apuntado en la Khan Academy), los objetos digitales educativos o los objetos de aprendizaje por sus propiedades.

iii Modalidad competencial, sin lugar a dudas, guiada por los principios del proyecto Tuning.

iv Modalidad en el método del programa, esto es, conforme a un conjunto de Normas de Alfabetización en información ( $A C R L, C A U L$, ANZIIL, etc.), que establecen qué enseñar en el programa.

v Modalidad en el modelo del programa (Big Six, Kulthau, Gavilán, etc.), que establece el cómo enseñar en el programa.

b)Diseño curricular

Por la propia naturaleza de las competencias en información, el programa debe ser escalable, lo que significa estar constituido por módulos, independientes entre sí, pero interconectados, de modo que cada curso o educando vaya decidiendo su progreso competencial, desde las destrezas, a las habilidades y competencias.

Esta propiedad escalable descansa, además, en la propia evolución de la aplicación de las competencias en información en programas formativos: los educandos deben comenzar a dominar las destrezas y habilidades informáticas (competencias digitales), que sólo tienen su verdadero sentido si luego conectan con el desarrollo de destrezas, habilidades y competencias en el procesamiento, uso, difusión y edición de la información al conocimiento (competencias en información), que sólo son posibles si se han logrado antes las competencias digitales. A este fenómeno de unir inextricablemente ambas competencias se le ha dado en llamar CI2 (REBIUN, 2008). La investigación científica y su proyección en los currículos académicos, además, están abriendo un campo sumamente interesante en la data literacy, con unas perspectivas formativas muy importantes (CALZADA;MARZAL, 2013).

En este mismo sentido se ido abriendo, muy recientemente, la noción de madurez digital e informacional como modo de medir y evaluar la forma cómo se incorporan los saberes, su normalización para establecer lo que debe saber y saber hacer, y su apropiamiento, esto es, la incorporación de los saberes a partir de beneficios, según los caracteres de los individuos u organizaciones. Normalización y apropiamiento se manifiestan como fenómenos complementarios: las normas deben flexibilizarse para su aplicación en situaciones concretas de saberes digitales e informacionales, en tanto el apropiamiento tecnológico debe considerar modelos de referencia a alcanzar conforme a prioridades específicas. La Web Index (WORLD WEB FOUNDATION, 2012), en un paso más, ha introducido los conceptos de "readiness" (infraestructura 
institucional y física), "usage" (contenidos) e "impact" (valor social, político y económico), como elementos susceptibles de medir en grado de madurez por apropiamiento de competencias digitales e informacionales.

\section{Metodología}

El marco conceptual, las convenciones científicas (incluso terminológicas) y la definición del campo de aplicación y tópico, concedían la fundamentación epistemológica suficiente al proyecto. Quedaba planteado, pues, un referente modélico al que dirigir las propuestas para diseñar programas de competencias y alfabetización en información, en principio desde las bibliotecas universitarias (GÓMEZ HERNÁNDEZ, 2010), como medio para apoyar la excelencia de las universidades en un modelo educativo competencial.

De otro lado se formulaban con solidez la hipótesis (integración de la educación en competencias informáticas e informacionales mediante programas de alfabetización en información para el modelo educativo de las universidades del siglo XXI), el objeto general (plantear el diseño instruccional y curricular de los programas de alfabetización en información y los beneficios de las competencias en información para la excelencia en universidades), objetivos específicos (derivados de la definición y enunciación de las fases que contempla el proyecto para efectuar un estudio de campo preliminar que garantice la implantación e implementación del programa de alfabetización en información), las fases (coherentes con un estudio de campo, esto es, reconocimiento de factores necesarios para desarrollar el programa en la universidad, identificación de agentes críticos y de grupos meta, formulación de una encuesta, su ejecución, procesamiento y análisis) y las fuentes de datos e información (la encuesta).

El proyecto planteaba también sus límites. No cabe duda que el diseño instruccional y curricular de un programa de alfabetización en información desde bibliotecas es un factor de innovación importante. Inspirados en experiencias comparables (propuestas de transformación de bibliotecas universitarias en CRAI), el proyecto optó por limitar su acción investigadora a un trabajo de campo preparatorio (fundamental y necesario) para conocer cómo se entendían las competencias en información, qué buenas prácticas ya existían y cómo se llevaban a cabo en el currículo de la universidad, cuál es el grado de conocimiento y posible compromiso de los agentes críticos en la integración de un programa de alfabetización en información. Por ello, el proyecto se dirigió a un número muy representativo de bibliotecas universitarias chilenas, que ha permitido tener un espectro muy sólido de la situación y así servir para un desarrollo futuro del proyecto: plantear un modelo de integración y desarrollo de programas de alfabetización en información para las universidades chilenas, como factor de excelencia y progreso en los rankings internacionales.

En el marco del proyecto de investigación, el Sistema de Bibliotecas de la Pontificia Universidad Católica de Chile diseñó, ensayó y luego 
procedió a someter una encuesta a los directores de bibliotecas de universidades públicas y privadas de Chile, con el fin de realizar un diagnóstico del estado del arte y nivel de desarrollo programático respecto a la formación en competencias informacionales o alfabetización en información (ALFIN) de sus respectivas universidades. La encuesta formulaba preguntas específicas referidas a la terminología empleada en estos programas, la definición de los conceptos a impartir contemplados en el diseño instruccional del programa, los estándares y modelos que definían el diseño pedagógico del programa y así detectar su compatibilidad con otros programas a escala nacional y sobre todo internacional, el programa didáctico con sus actividades, niveles y modalidades de implementación, así como los tipos y herramientas de evaluación. El objetivo fundamental es que esta información pueda ser pertinente para que las instituciones educativas tengan un referente básico para iniciar, mejorar y/o ampliar el desarrollo de estas competencias en sus respectivas organizaciones.

Para llevar a cabo los objetivos del proyecto se revisó el sitio del Consejo Nacional de Educación para identificar el nombre de los directores, correo electrónico y URL de 41 bibliotecas universitarias (Anexo 1). Posteriormente se les envió una encuesta elaborada con la aplicación Google Docs.

La encuesta de 19 preguntas cerradas y una pregunta abierta incluye: cinco preguntas relacionadas con datos de la biblioteca y de la persona que la contesta; a partir de la pregunta 6 se recaba información sobre el desarrollo de las competencias informacionales. La última pregunta recoge observaciones o comentarios (Anexo 2).

Se recibieron 24 respuestas que representan el 58,53\% de las bibliotecas encuestadas, 14 de las cuales corresponden a bibliotecas de Universidades pertenecientes al Consejo de Rectores de las Universidades Chilenas ( $\mathrm{CRUCH}$ ), lo que significa que es una muestra representativa para los fines de la investigación.

\section{Resultados y discusión}

Obviando las cinco primeras preguntas, relativas a la identificación del encuestado, a efectos de la investigación se hacen especialmente relevantes las respuestas a partir de la sexta pregunta y la interpretación más plausible, según procedemos a analizar.

Pregunta 6. ¿Qué nombre asigna su institución a la alfabetización informacional?

Se ofrecían 8 alternativas, de las cuales se podía seleccionar más de una y/o se podía agregar otra. Entre las opciones de términos también se registraban los siguientes acrónimos: ALFIN (entendido como alfabetización informacional o alfabetización en información), DCI (desarrollo de competencias informacionales o desarrollo de competencias en información) y DHI (desarrollo de habilidades informacionales o 
desarrollo de habilidades informativas o desarrollo de habilidades en información).

Los términos seleccionados más usados y en el mismo porcentaje (26\%) corresponden a ALFIN, competencias informacionales y desarrollo de competencias informacionales. Le siguen en orden decreciente: alfabetización informacional (21\%), desarrollo de competencias informativas $(16 \%)$, competencias en información $(11 \%)$ y DCI $(11 \%)$. Otras denominaciones utilizadas son habilidades informacionales, uso de recursos de información electrónica, programa de educación en información, programa para la formación de competencias informacionales y educación de usuarios. Hay instituciones, hasta un número de 4, que no le dan un nombre. Estos resultados reflejan que existe una variedad de términos para denominar estas competencias.

Pregunta 7. ¿Tiene implementado un Programa de ALFIN en su institución? y Pregunta 8. ¿Realiza actividades de ALFIN?, que podemos considerar agrupadas, por buscar en qué medida la institución hace relevante este tipo de formación, como un programa o un elenco de actividades.

En el primer caso implicaba que la institución ha desarrollado y ofrece un programa que incluye: misión, objetivos, recursos físicos, humanos y tecnológicos, contenidos, metodología y evaluación. El 42\% respondió afirmativamente. La segunda pregunta se refiere a sesiones que abarcan entre 1-10 horas. La mayoría de las universidades (83\%) contestó afirmativamente.

Pregunta 9. ¿Qué cantidad de horas abarca la actividad de ALFIN?

Esta pregunta ofrecía 4 opciones y se podía seleccionar más de una. La mitad de las instituciones, doce, seleccionó 1 a 3 horas (57\%), seguida por la selección de 3 a 6 horas (29\%), luego 6 a 10 horas (29\%) $y$, por fin en cuatro casos, más de 10 horas (19\%).

Pregunta 10. ¿Cuál es la definición de ALFIN que sigue su institución?

De forma inducida se ofrecieron las definiciones de la $A L A / A C R L$, CILIP, CAUL, Webber y Johnston y Bruce. En general, las instituciones que realizan actividades de ALFIN se basan en definiciones emanadas de asociaciones profesionales 0 expertos internacionalmente reconocidos tales como: ALA-ACRL (American Library Association-Association of College and Research Libraries) de Estados Unidos, CILIP (Chartered Institute of Library and Information Professionals) de Gran Bretaña, CAUL (Council of Australian University Librarians) de Australia. Los expertos Sheila Webber y Bill Johnston de Gran Bretaña y Christine Bruce de Australia, entre otros, también han tenido influencia en esta área. 
La definición más usada, la mitad de las instituciones chilenas, es la de la ALA-ACRL (50\%), seguida por la de CILIP (17\%) y CAUL (17\%). Ninguna institución eligió la definición de Webber y Johnston ni la de Bruce. Otras definiciones incluidas por 2 de las bibliotecas encuestadas son:

1 "Conjunto de procedimientos, conceptos y valores necesarios para resolver problemas que impliquen la búsqueda, selección, organización, análisis y comunicación de la información" (Gómez Hernández, 2000).

2 "Habilidad para buscar, procesar y analizar información procedente de diversas fuentes. Extrae, relaciona, interpreta, organiza y presenta información, lógica y comprensiblemente sobre diversos temas, situaciones y problemas, evidenciando una construcción personal de ideas". (No se consigna fuente).

Pregunta 11. ¿En qué normas/modelos se basa la institución para desarrollar su programa o actividades de ALFIN?

Los modelos y las normas, también están elaborados por asociaciones de bibliotecarios y/o expertos, con el objetivo de establecer cuáles son las competencias y cómo se deben implementar. En la encuesta se ofrecen varias opciones y las personas pueden seleccionar más de una opción: Modelo británico e irlandés SCONUL (Society of College, National and University Libraries), Modelo Big6 de los educadores americanos Mike Eisenberg y Bob Berkowitz, Modelo de la experta estadounidense Carol Kuhlthau y el Modelo relacional de la australiana Christine Bruce, Normas americanas de la ACRL (Association of College and Research Libraries), Normas CAUL (Council of Australian University Librarians) de Australia, Normas australianas y neozelandesas ANZIIL (Australian and New Zealand Institute for Information Literacy). La mayoría de las bibliotecas seleccionó las normas de la ACRL (86\%), seguida por Modelo SCONUL (29\%), Modelo Big6 (21\%), Normas CAUL (14\%). Ninguna institución seleccionó: Normas ANZIIL, Modelo Kuhlthau ni el Modelo relacional de Bruce.

Pregunta 12. ¿En qué niveles está implementado el programa o actividades de ALFIN?, Pregunta 13. ¿Cuál es la modalidad de oferta? y Pregunta 14. ¿Cuál es la modalidad de instrucción con la que realizan las actividades de ALFIN?

Para la pregunta 12 se ofrecían 3 alternativas y las personas podían contestar más de una. Todas las instituciones que tienen un programa u ofrecen actividades de ALFIN lo han implementado en el pregrado $(100 \%)$ y también a los docentes (76\%) y postgrados (67\%). Se mencionan otros niveles que hacen referencia a su orientación al personal administrativo de la universidad y de la biblioteca, y los antiguos alumnos o egresados. 
Para la pregunta 13 se proponían cuatro alternativas y las personas podían seleccionar más de una. Los resultados son: sesiones solicitadas por docentes en sus cursos ( $86 \%)$, sesiones independientes (55\%), un curso integrado a la malla curricular (36\%) y en un curso que ofrece la universidad (14\%)

También para la pregunta 14 se ofrecían siete alternativas y las personas podían seleccionar más de una. Los resultados son: presencial $(82 \%)$, grupal $(77 \%)$, taller $(73 \%)$, práctica $(50 \%)$, individual $(32 \%)$, teórica $(27 \%)$, en línea (9\%). No se indican otras modalidades. realizan?

Pregunta 15. ¿De qué carácter son las actividades de ALFIN que

Se presentaban una opción, obligatorio u optativo, y se podía seleccionar ambas. La mayoría ( $86 \%$ ) seleccionó, valga la redundancia, la opción optativa.

Pregunta 16. ¿Evalúa el programa/actividades de ALFIN?, Pregunta 17. ¿Qué evaluación aplica?, Pregunta 18. ¿Qué aspectos de la actividad evalúa?, Pregunta 19. ¿Qué herramientas utiliza para evaluar el programa/sesión/ actividad?

En la pregunta 16 la mayoría respondió afirmativamente (71\%) y sólo un $21 \%$ respondió negativamente. La pregunta 17 ofrecía tres alternativas y las personas podían seleccionar más de una: en igual porcentaje afirmaron el uso de la evaluación sumativa (61\%) y la evaluación formativa $(61 \%)$, el menor porcentaje correspondió a la evaluación diagnóstica (39\%).

La pregunta 18 planteaba seis opciones y se podía seleccionar más de una, siendo sus resultados: logro de objetivos $(79 \%)$, metodología usada $(74 \%)$, contenidos $(68 \%)$, recursos tecnológicos $(58 \%)$, relator $(47 \%)$ y sala $(37 \%)$.

Por fin, la pregunta 19 ofrecía seis opciones, con posibilidad de seleccionar más de una: la encuesta es la más popular (53\%), seguida por cuestionario (32\%), observación (32\%), focus group (16\%), entrevistas $(16 \%)$, portafolio $(16 \%)$. Entre otras herramientas algunas bibliotecas mencionan: rúbrica, informe de avance de investigación colaborativos (los cuales tienen que ser defendidos y presentados al final del programa).

Los resultados muestran a las claras la importancia de definir el significado de la alfabetización en información para saber cómo desarrollarla, si se logra o no este desarrollo, así como también contar con normas y modelos que indiquen cuál es el diseño instructivo y modelo curricular mejores para asegurar que se alcanzan los objetivos de aprendizaje. Entre las bibliotecas universitarias de Chile que respondieron la encuesta, no existe un consenso en la concepción para designar sus actividades de desarrollo de competencias informacionales ya que le asignan distintos términos e incluso coexiste más de uno en la misma institución. Esta variedad terminológica es común en todos los países hispanoamericanos. 
Los resultados de la encuesta ponen de manifiesto la influencia, especialmente, del modelo americano a través de la definición y normas emanadas de la ALA-ACRL para desarrollar las actividades y/o programa. En menor medida se nota la influencia australiana de SCONUL.

Es interesante mencionar que de las 12 bibliotecas que eligieron la definición de la ALA-ACRL, sólo 7 también siguen las normas/estándares de ACRL, y las otras 5 usan sus normas en combinación con otros estándares o modelos: CAUL/Big 6, solo Big 6 y Big 6/SCONUL. Dos no seleccionaron ningún estándar. De las 4 instituciones que seleccionaron la definición de CAUL, una de ellas usa las normas/estándares ACRL y SCONUL, una usa normas ACRL y dos no seleccionaron ninguna norma. Tres instituciones seleccionaron la definición de CILIP, una de ellas seleccionó el modelo SCONUL, una agregó la definición de Gómez Hernández y las normas de ACRL en conjunto con SCONUL y dos de ellas no seleccionaron ninguna norma o estándar. Dos instituciones enuncian definiciones propias, una de ellas selecciona la norma ACRL y CAUL, la segunda no selecciona ninguna norma. Cabe destacar que en todas las universidades que realizan la actividad, los alumnos de pregrado reciben la capacitación en ALFIN y también hay una tendencia positiva en ese sentido para los docentes y alumnos de postgrado. La mitad de las instituciones dedican entre 1-3 horas.

Hay una tendencia hacia la capacitación presencial, grupal y a través de talleres. Se realiza evaluación sumativa y formativa, destacándose la evaluación del logro de los objetivos y metodología, en menor porcentaje se evalúa los contenidos. La encuesta es la herramienta evaluativa más utilizada. Se observa que aunque hay un importante avance en las universidades encuestadas, también se notan diferentes niveles de desarrollo y que hay que seguir mejorando la capacitación en ALFIN.

A la luz de estos resultados, parece necesario:

a)Seguir mejorando la capacitación en ALFIN con el propósito de nivelar la implementación de actividades en cada institución para lo cual se mencionan algunas recomendaciones.

b)Contar con un programa que reúna características de las mejores prácticas (misión, objetivos, recursos físicos, humanos y tecnológicos, contenidos, metodología y evaluación) contribuye al desarrollo, evaluación y mejoramiento de la implementación exitosa de ALFIN, por lo que se recomienda elaborar un programa estructurado a las instituciones que todavía no lo tienen.

c)Buscar estrategias conjuntas con los docentes que promuevan incorporar el ALFIN dentro de los programas de los cursos, de manera que la actividad llegue a ser obligatoria para que todos los alumnos tengan la oportunidad de desarrollar la capacidad de buscar, evaluar, gestionar y usar información durante su carrera y posterior vida profesional.

d) Prestar especial atención a los alumnos de postgrado que requieren poseer esas competencias para apoyar sus investigaciones desde el inicio de sus estudios. 
e)Evaluar todas las actividades de ALFIN que se realicen así como aplicar diversas herramientas evaluativas para asegurar el logro de los objetivos de aprendizaje.

f) Contar con el apoyo de las autoridades de cada Universidad y sus respectivas Facultades, para que el desarrollo de las competencias informacionales se convierta en un objetivo transversal en la formación de todos los alumnos y forme parte de los métodos de enseñanza que promueven un aprendizaje activo y profundo.

\section{Conclusiones}

Las universidades deben experimentar una radical transformación en su modelo como organización, pero desde luego en su modelo educativo. No sólo debe enfrentar los desafíos de la Sociedad del conocimiento, sino que debe responder con celeridad a una nueva Educación que garantice la excelencia en los conocimientos de los egresados y también su capacidad en el aprendizaje permanente, en la innovación. Estas exigencias, junto con un progreso evidente en las TIC hacen que la conocida división en Educación Formal, No Formal e Informal sea cada vez más irrelevante: el e-learning, m-learning, los MOOC son la manifestación de una Educación del siglo XXI. Los educandos, además, han variado y con ellos la comunidad universitaria: ya no son sólo, pronto ni siquiera mayoritariamente, alumnos de bachillerato, sino serán alumnos procedentes de otros países y sistemas educativos (por la movilidad), profesionales en constante actualización, la tercera edad (universidad para mayores). Por último, dentro del auge incontestable de una cultura de la evaluación, las universidades deben ser evaluadas, acreditadas y certificadas, ordenadas en rankings por excelencia académica, de lo que derivará su financiación, su implantación y su supervivencia.

Estos desafíos tienen, sin duda, una respuesta inmediata y en un modelo educativo competencial, pero este modelo no debe reducirse a unas propuestas pedagógicas y aplicaciones didácticas, con ser esenciales. Se hace imprescindible definir qué es exactamente competencia (con formulación de un modelo, tipos, prácticas, proyecto metodológico) y luego competencia educativa: es irrenunciable conocer con certeza el qué para articular el cómo.

Si la formulación del modelo educativo por competencias debe corresponder al ámbito de la filosofía educativa de una universidad, las competencias en información y su apropiación mediante programas de alfabetización en información deben corresponder al ámbito práctico de definición del currículo de los estudios en la universidad desde Grado a Postgrado y Doctorado. Un programa de alfabetización en información responde con eficacia y contundencia a todas y cada una de las transformaciones que arriba hemos anotado que experimenta la Educación, con un doble efecto inmediato y muy saludable: un progreso en las competencias profesionales de los egresados (un indicador fundamental), pero también un factor de progreso en los rankings para la universidad, como entidad educativa. 
La relevancia de los programas de alfabetización en información, pues, obliga a ser muy cuidadosos en su diseño instruccional y curricular. Debe estar bien asumido por la comunidad universitaria, bien formulado en los planes y programas de actuación de las universidades, bien visible en su reconocimiento académico mediante créditos y un sistema de evaluación propio, debe implantarse como un programa que no esté aislado dentro de una universidad, sino con plena interoperabilidad con los programas de otras universidades nacionales y de otros países, una meta regional (latinoamericana) o mundial, según se observa en la tendencia del modelo de universidad actual.

Sin duda, estos han sido los objetivos de este proyecto preliminar, destinado a plantear una propuesta de modelo de integración curricular en universidades chilenas de programas de alfabetización en información, con una vocación inmediata de colaborar en la creación de un referente nacional, enseguida latinoamericano y luego global.

\section{Referencias}

ALONSO, C.; GALLEGO, D. Los estilos de aprendizaje como competencias para el estudio, el trabajo y la vida. Revista de Estudios de Aprendizaje, v. 6, n. 6, 2010. Disponible en: <http://www.uned.es/revistaestilosdeaprendizaje>. Acceso: 4 abril 2014

BRUCE, C. S. The seven faces of information literacy. Adelaida: Auslib Press, 1997.

CALZADA, F. J.; MARZAL, M. A. Incorporating data literacy into information literacy programs: core competencies and contents. Libri, v. 63, n. 2, p. 123-134, 2013.

CARRERA, C.; MARÍN, R. Modelo pedagógico para el desarrollo de competencias en educación superior. Revista Actualidades investigativas en Educación, v. 11, n. 1, p. 1-32, 2011.

GARCÍA RETANA, J. A. Modelo educativo basado en competencias: importancia y necesidad. Revista Actualidades investigativas en Educación, v. 11, n. 3, p. 1-24, 2011.

GÓMEZ HERNÁNDEZ, J. A. Las bibliotecas universitarias y el desarrollo de las competencias informacionales en los profesores y los estudiantes. Revista de Universidad y Sociedad del Conocimiento (RUSC), v. 7, n. 2, p. 39-49, 2010. Disponible en: <http://rusc.uoc.edu/ojs/index.php/rusc/article/view/v7n2-gomez/v7n2-gomez. > .

Acceso: 4 abril 2014.

JONNAERT, P. et al. Revisión de la competencia como organizadora de los programas de formación: hacia un desempeño competente. Ginebra: Oficina Internacional de Educación BIE/UNESCO, 2006.

LUENGO, J.; LUZÓN, A.; TORRES, M. Las reformas educativas basadas en el enfoque por competencias: una visión comparada. Profesorado. Revista de Currículum y Formación del Profesorado, v. 12, n. 3, p. 1-10, 2008. 
MARZAL, M. A.; PARRA, P. Bibliotecas integradas: alfabetización en información como estímulo de cooperación. El Profesional de la Información, v. 19, n. 5, p. 463-468, septiembre-octubre 2010.

MULDER, M.; WEIGEL, T.; COLLINS, K. The concept of competence in the development of vocational education and training in selected EU member states: a critical analysis. Journal of Vocational Education and Training, v. 59, p.67-88, 2007.

PERRENOUD, P. Diez nuevas competencias para enseñar. México: SEP, 2004.

REBIUN. Grupo de trabajo ALFIN. Guía de buenas prácticas para el desarrollo de las competencias informacionales en universidades españolas. Madrid: CRUE-TIC, 2008. Disponible en: $<$ http://www.rebiun.org/opencms/opencms/handle404?exporturi=/export/docReb/gui a buenas practicas.doc\&\%5d>. Acceso: 4 abril 2014.

RIAL, A. Diseño curricular por competencias: el reto de la evaluación. Jornades d'avaluació dels aprenenatges a partir de competències. Girona: Universitat, 2007. Disponible en: <http://hdl.handle.net/10256/819>. Acceso: 4 abril 2014.

ROEGIERS, X. Curricular reforms guide Schools: but, where to? Prospects, v. 37, n. 2, p. 155-186, 2007.

TARDIF, J. Développer un programme par compétences: de l'intention à la mise en oeuvre. Pédagogie collégiale, v. 16, n. 3, p. 36-45, 2003.

WEBBER, S.; JOHNSTON, B. Information literacy in higher education: a review and case study. Studies in Higher Education, v. 28, n. 3, p. 335352, 2003.

WORLD WIDE WEB FOUNDATION, Web Index 2012, 2012. Disponible en: $<$ http://thewebindex.org/2012/10/2012-Web-Index-Key-Findings.pdf>. Acceso: 4 abril 2014. 